\title{
Characteristics of Patients with Upstaging by Sentinel Lymph Node Biopsy of the No Neck in Head and Neck Cancer
}

\author{
Didier Dequanter $^{1 *}$, Philippe Lothaire ${ }^{2}$ \\ ${ }^{1}$ Maandal, Alsemberg, Belgium; ${ }^{2}$ Rue de Gozée, Montigny le tilleul, Belgium. \\ Email: didier.dequanter@pandora.be
}

Received February 15 ${ }^{\text {th }}, 2011$; revised May 9 $9^{\text {th }}, 2011$; accepted May $17^{\text {th }}, 2011$.

\begin{abstract}
Introduction: To investigate the possible role of sentinel lymph node biopsy (SLNB) to upstage the N0 neck in patients with oral and oropharyngeal squamous cell carcinoma. Methods: Patients with T1-T2 oral and oropharyngeal squamous cell carcinoma accessible to injection and staged $N 0$ into the neck by palaption and CTscan were enrolled in the study. All patients underwent regular follow-up to identify possible recurrence. Results: A sentine lymph node biopsy was performed by 21 consecutive patients. 4 of the 21 patients were upstaged by SNLB. There was a mean follow-up of 31 months. Two patients developed subsequent disease after having been staging by SLNB, respectively negative in one case and positive in the other case. Tumor site, the staging of the primary tumor, presence of ulceration, tumor thickness were the same in the upstaged initially N0 patients. Conclusions: Sentinel lymph node biopsy can be used to upstage the NO neck patients in perhaps well defined patients.
\end{abstract}

Keywords: Sentinel Lymph Node, Characteristics of Patients, Head and Neck Cancer

\section{Introduction}

Management of the N0 neck in head and neck squamous cell carcinoma is an important issue for the head and neck surgeon. Furthemore, in patients with head and neck squamous cell carcinoma, the presence of lymph node metastases is the most prognosticator.

Sentinel lymph node biopsy originally was described as a means of identifying lymph node metastases in malignant melanoma and breast carcinoma. The use of SLN biopsy in patients with oral and oropharyngeal squamous cell carcinoma N0 necks was investigated to determine whether the pathology of the SLN reflected that of the neck. Most studies [1-3] showed that sentinel lymph node localization is technically feasible in head and neck surgery and is predictive of cervical metastasis. The second step was to demonstrate that the sentinel lymph node biopsy can be used to upstage the N0 neck in patients with early sublinical nodal disease. The aim of our prospective study (part of EORTC protocol 24021) was to confirm the possible role of sentinel lymph node biopsy alone to upstage the clinically N0 neck in patients with oral and oropharyngeal squamous cell carcinoma and to determine the characteristics of such patients for perhaps a more selected role of the technique.

\section{Methods}

Patients undergoing elective neck dissections for T1 - T2 head and neck squamous cell carcinoma accessible to injection were enrolled into our study. The technique of sentinel node biopsy involves the combination of mapping the main lymph nodes fields of the neck by a radioactive tracer. The tracer passes along the lymphatics channels to accumulate in the sentinel nodes making them radioactive. At surgery, the nodes are identified by gamma probe and harvested for histological examination. A neck dissection completed the surgical procedure and the pathology of the SLN was compared with that of the remaining neck dissection. The nodes were fixed in $10 \%$ neutral buffered formalin and after fixation bisected through the hilum, if this was identifiable, or through the long axis of the node. If the thickness of the halves was more than $2.5 \mathrm{~mm}$, the slices were further sectioned to provide additional $2.5 \mathrm{~mm}$ thick blocks. Two histological sections should be taken from each $2.5 \mathrm{~mm}$ slice one to prepare for $\mathrm{H} \& \mathrm{E}$, the other for cytokeratin antibody and the remaining four for further evaluation if needed. 


\section{Results}

An sentinel lymph node (SLN) biopsy was performed by 21 patients (median age: 63 years). Of the 21 patients, 5 were women and 16 were men. The primary tumor was located on the oral tongue in 6 cases, at the Floor of the mouth in 10 cases and at the oropharynx in 5 cases. Eight primary tumors were stage $\mathrm{T} 1$. All others tumors were stage T2 (Table 1). All the tumors were clinically staged cN0 by palapation and CT scan or MRI. Neck dissection was carried out in all patients. One patient with a midline tumor underwent SLNB on the controlateral side. During neck dissection, SLN was removed and identified seperately. SLNs were identified in all patients and accurately predicted the pathological nodal status in 20 of the 21 patients. Three patients had positive SLNs at multiple neck levels. 4 of the 22 patients were upstaged by SNLB. There was a mean follow up of 31 months. Two patients developed susbsequent disease after having been staging respectively negative in one case and positive in the other case by SNLB. The tumor site ( posterieur part of the mobile tongue), the staging of the primary tumor (T2), presence of ulceration, tumor thickness (superior to $5 \mathrm{~mm}$ ) were the same in the upstaged patients clinically N0.

\section{Discussion}

Management of the N0 neck in head and neck squamous cell carcinoma is an important issue for the head and neck surgeon. In patients with head and neck cancer, the presence of lymph nodes metastases is the most important factor. Our study comfirmed as the most studies [1-9] that sentine lymph node biopsy(SLNB) is an accurate reflector of the status of the regional lymph nodes [1,3]. Furthermore, SLNB is technically feasible and the tech- nique is safe. The sentinel lymph node technique offered a more accurate staging and mapping for the lymphatic drainage, has the potential to decrease the number of neck dissections performed. The potential for minimally invasive surgery based on this technology certainly exists.

But as new all techniques, the SLNB is gaining popularity and it will be important to focus on refinements of technique. The majority of teams used SLNB for T1 and T2 [2]. In his series, Taylor comfirmed that sentinel lymph node localization is technically feasible in head and neck surgery and is predictive of cervical metastasis.

Some authors used the technique for T2 to T4 N0 head and neck patients. In his series, Nieuwehuis et al. [4] treated 22 patients with T2 to T4 N0 oral and oropharyngeal squamous cell carcinoma. The sensitivity of the SN procedure for predicting lymph nodes metastases was 89\% when SN was identified by scintigraphy and found in the specimen. The overall accuracy of the SN procedure for predicting the presence or absence of lymph nodes metastases in the neck was 95\%. They validate the SN hypothesis for oral and oropharyngeal cancer but SN identification and thus biopsy does not seem to be reliable in patients with tumors located in or close to the midline.

Some authors used the SLNB technique to detemine the surgical treatment of the controlateral neck patients with N2a, N2b and N3 neck disease [6]. In the attempt to etablish the reliability of extended use of the SLNB technique, 100 consecutive untreated patients with tumors located in the oral cavity, oropharynx, hypopharynx and larynx entered their study. In the N+ Group, no metastases were found in the sentinel nodes on the controlateral

Table 1. Characteristics of patients treated by SLNB.

\begin{tabular}{|c|c|c|c|c|c|c|c|c|}
\hline Patients & Age & Tumor Site & $\mathrm{cT}$ & $\mathrm{cN}$ & SLN(n) & Pos Nodes ND & Localisation & Type ND \\
\hline 1 & 56 & Tongue & cT1 & cN0 & 1 & 0 & II & $\mathrm{II}+\mathrm{III}+\mathrm{V}$ \\
\hline 2 & 65 & Floor of the mouth & cT2 & cNO & 2 & 0 & II & $\mathrm{I}+\mathrm{II}+\mathrm{III}+\mathrm{V}$ \\
\hline 3 & 67 & Tongue & cT2 & cNo & 1 & 1 & II & $I+I I+I I I+V$ \\
\hline 4 & 71 & Tongue & cT2 & cNo & 3 & 3 & $\mathbf{I}+\mathbf{I I}$ & $I+I I+I I+V$ \\
\hline 5 & 67 & Tongue & cT2 & cNo & 3 & 1 & $I I+I I I+V$ & $\mathbf{I I}+\mathbf{I I I}+\mathbf{V}$ \\
\hline 6 & 51 & Tongue & cT2 & cNO & 2 & 1 & II + IV & $I I+I I I+I V+V$ \\
\hline 7 & 70 & Floor of the mouth & cT2 & cNO & 2 & 0 & III & $\mathrm{II}+\mathrm{III}+\mathrm{V}$ \\
\hline 9 & 62 & Floor of the mouth & cT2 & cNO & 1 & 0 & I & $\mathrm{I}+\mathrm{II}+\mathrm{III}+\mathrm{IV}$ \\
\hline 10 & 64 & Oropharynx & cT1 & cNO & 0 & 3 & II & $\mathrm{II}+\mathrm{III}+\mathrm{IV}+\mathrm{V}$ \\
\hline 11 & 64 & Oropahrynx & cT2 & CNO & 1 & 0 & $\mathrm{~V}$ & $\mathrm{~V}$ \\
\hline 12 & 51 & Floor of the mouth & cT2 & cN0 & 1 & 0 & II & II + III \\
\hline 13 & 51 & Floor of the mouth & cT1 & cNO & 3 & 0 & II & II + III \\
\hline 14 & 52 & Oropahrynx & cT1 & cNO & 1 & 0 & II & \\
\hline 15 & 73 & Oropharynx & cT2 & cNO & 1 & 0 & II & \\
\hline 16 & 79 & Oropharynx & cT1 & CNO & 1 & 0 & & \\
\hline 18 & 46 & Floor of the mouth & cT1 & cNO & 1 & 0 & III & $\mathrm{II}+\mathrm{III}+\mathrm{V}$ \\
\hline 19 & 54 & Floor of the mouth & cT1 & cNO & 2 & 0 & II & $\mathrm{I}+\mathrm{II}+\mathrm{III}$ \\
\hline 20 & 63 & Floor of themouth & cT1 & cNO & 1 & 0 & II & $\mathrm{I}+\mathrm{II}+\mathrm{II}$ \\
\hline 21 & 58 & Floor of themouth & cT2 & cNO & 2 & 0 & II & $\mathrm{I}+\mathrm{II}+\mathrm{III}$ \\
\hline
\end{tabular}


neck of 21 patients and also the remaining nodes were negative; in four patients, metastases were found only in sentinel nodes. In 16 patients, lymphoscintigraphy did not identify any sentinel node and histology of all lymph nodes was negative for metastases. The SLNB technique may likely be used also in T3 and T4 oro-hypopharyngeal and laryngeal primary tumors and to determine surgical treatment of the controlateral neck in patients with N2a, N2b and N3 neck disease.

In fact, the management of the N0 neck in oral and oropharyngeal cancer is often determined by the risk of metastases related to features of the primary tumor. When the risk of metastases is $>20 \%$, elective neck dissection has been advocated. Depsite the number of publications, only a few articles have been published that discuss the relation between histological predictors and the risk of cervical metastases and so perhaps a better selection of patients candidates to SLNB. O' Brien, in his series [10], showed that selective treatment of the clinically negative neck based on the primary tumor site and stage led to high rate of regional disease control. Ross et al. [8] showed that tumor thickness, perineural invasion were all histological predictors for cervical metastases. This report analyses the results of our treatment's experience. All the N0 head and neck patients upstaged by the technique had the same histopathological characteristics of the primary tumor. All the patients presented at diagnosis a ulcerated tumor staged T2, located at the mobile part of the tongue with a tumor thickness superior to $5 \mathrm{~mm}$. Our preliminary investigation suggests the importance of analysing the characteristics of the primary tumor when the SLNB technique is selected in clinically N0 head and neck patients. Indeed, new studies need to focus on refinements of selection of the SLNB technique.

\section{Conclusions}

Sentinel lymph node biospy can be used to upstage the N0 neck patients in perhpas well define patients.

\section{REFERENCES}

[1] T. Shoaib, D. S. Soutar, D. G. MacDonald, I. G. Camilleri, D. J. Dunaway, H. W. Gray, G. M. McCurrach, R. G. Bessent, T. I. MacLeod and A. G. Robertson, "The Accuracy of Head and Neck Carcinoma Sentinel Lymph Node Biopsy in the Clinically N0 Neck," Cancer, Vol. 91, No. 11, 2001, pp. 2077-2083.
doi:10.1002/1097-0142(20010601)91:11<2077::AID-CN CR1235>3.0.CO;2-E

[2] R. J. Taylor, R. L. Wahl, P. K. Sharma, C. R. Bradford, J. E. Terrell, T. N. Teknos, E. M. Heard, G. T. Wolf and D. B. Chepeha, "Sentinel Node Localization in Oral Cavity and Oropharynx Squamous Cell Cancer," Archives of Otolaryngology—Head \& Neck Surgery, Vol. 127, No. 8, 2001, pp. 970-974.

[3] W. M. Koch, M. A. Choti, A. C. Civelek, D. W. Eisele and J. R. Saunders, "Gamma Probe-Directed Biopsy of the Sentinel Node in Oral Squamous Cell Carcinoma," Archives of Otolaryngology-Head \& Neck Surgery, Vol. 124, No. 4, 1998, pp. 455-459.

[4] E. J. Nieuwenhuis, I. van der Waal, C. R. Leemans, A. Kummer, R. Pijpers, J. A. Castelijns, R. H. Brakenhoff and G. B. Snow, "Histopahtologic Validation of the Sentinel Node Concept in Oral and Oropharyngeal Squamous Cell Carcinoma,” Head Neck, Vol. 27, 2005, pp. 150-158. doi:10.1002/hed.20126

[5] K. T. Pitman, J. T. Johnson, M. L. Brown and E. N. Myers, "Sentinel Lymph Node Biopsy in Head and Neck Squamous Cell Carcinoma,” Laryngoscope, Vol. 112, No. 12, 2002, pp. 2101-2113. doi:10.1097/00005537-200212000-00001

[6] L. Barzan, S. Sulfaro, F. Alberti, D. Politi, M. Pin, M. G. Savignanao, W. Marus, O. Zarcone and R. Spaziante, “An Extended Use of the Sentinel Node in Head and Neck Squamous Cell Carcinoma: Results of a Prospective Study of 100 Patients," Acta Otorhinolaryngologica Italica, Vol. 24, 2004, pp. 145-149.

[7] S. J. Stoeckli, "Sentinel Node Biopsy for Oral and Oropharyngeal Squamous Cell Carcinoma of the Head and Neck,” Laryngoscope, Vol. 117, 2007, pp. 1539-1551. doi:10.1097/MLG.0b013e318093ee67

[8] G. L. Ross, D. S. Soutar, D. G. Macdonald, T. Shoaib, I. G. Camilleri and A. G. Roberston, "Improved Staging of Cervical Metastases in Clinically Node-Negative Patients with Head and Neck Squamous Cell Carcinoma,” Annals of Surgical Oncology, Vol. 11, 2004, pp. 213-218. doi:10.1245/ASO.2004.03.057

[9] N. Rigual, W. Douglas, D. Lamonica, S. Wiseman, R. Cheney, W. Hicks and T. Loree, "Sentinel lymph Node Biopsy: A Rational Approach for Staging T2N0 Oral Cancer," Laryngoscope, Vol. 115, No. 12, 2005, pp. 2217-2220. doi:10.1097/01.mlg.0000187870.82699.ed

[10] C. J. O’Brien, S. J. Traynor, E. McNeil, J. D. McMahon and J. M. Chaplin, "The Use of Clinical Criteria Alone in the Management of the Clinically Negative Neck among Patients with Squamous Cell Carcinoma of the Oral Cavity and Oropharynx," Archives of Otolaryngology—Head \& Neck Surgery, Vol. 126, No. 3, 2000, pp. 360-365. 